(2) Open Access Full Text Article

\title{
Prevalence and characteristics of patients with
} low levels of low-density lipoprotein cholesterol
in northern Denmark: a descriptive study

This article was published in the following Dove Press journal:

Clinical Epidemiology

26 February 2015

Number of times this article has been viewed

\author{
Sigrun Alba Johannesdottir \\ Schmidt ${ }^{\prime}$ \\ Uffe Heide-Jørgensen' \\ Angelika D Manthripragada ${ }^{2}$ \\ Vera Ehrenstein' \\ 'Department of Clinical Epidemiology, \\ Aarhus University Hospital, Aarhus, \\ Denmark; ${ }^{2}$ Center for Observational \\ Research, Amgen Inc., Thousand Oaks, \\ CA, USA
}

Correspondence: Sigrun Alba Johannesdottir Schmidt Department of Clinical Epidemiology, Aarhus University Hospital, Olof Palmes Allé 43-45, 8200 Aarhus N, Denmark Tel +4587168245

Fax +4587167215

Emailsaj@clin.au.dk
Background: With the emergence of new lipid-lowering therapies, more patients are expected to achieve substantial lowering of low-density lipoprotein cholesterol (LDL-C). However, there are limited data examining the clinical experience of patients with low $(<1.3 \mathrm{mmol} / \mathrm{L})$ or very low $(<0.65 \mathrm{mmol} / \mathrm{L})$ levels of LDL-C. To provide information on patients with low LDL-C, we identified and characterized persons with low LDL-C using data from Danish medical databases

Methods: Using a population-based clinical laboratory database, we identified adults with at least one LDL-C measurement in northern Denmark between 1998 and 2011 (population approximately 1.5 million persons). Based on the lowest measurement during the study period, we divided patients into groups with low $(<1.3 \mathrm{mmol} / \mathrm{L})$, moderate $(1.3-3.3 \mathrm{mmol} / \mathrm{L})$, or high $(>3.3 \mathrm{mmol} / \mathrm{L})$ LDL-C. We described their demographic characteristics, entire comorbidity history, and 90-day prescription history prior to the lowest LDL-C value measured. Finally, we further restricted the analysis to individuals with very low LDL-C $(<0.65 \mathrm{mmol} / \mathrm{L})$.

Results: Among 765,503 persons with an LDL-C measurement, 23\% had high LDL-C, 73\% had moderate LDL-C, and 4.8\% had low LDL-C. In the latter group, 9.6\% ( $0.46 \%$ of total) had very low LDL-C. Compared with the moderate and high LDL-C categories, the low LDL-C group included more males and older persons with a higher prevalence of cardiovascular disease, diabetes, chronic pulmonary disease, ulcer disease, and obesity, as measured by hospital diagnoses or relevant prescription drugs for these diseases. Cancer and use of psychotropic drugs were also more prevalent. These patterns of distribution became even more pronounced when restricting to individuals with very low LDL-C.

Conclusion: Using Danish medical databases, we identified a cohort of patients with low LDL-C and found that cohort members differed from patients with higher LDL-C levels. These differences may be explained by various factors, including prescribing patterns of lipid-lowering therapies.

Keywords: cross-sectional study, hyperlipidemia, registries, statins

\section{Introduction}

Epidemiological studies have demonstrated a log-linear direct relationship between low-density lipoprotein cholesterol (LDL-C) concentration and cardiovascular disease risk. ${ }^{1,2}$ A review of primary and secondary intervention trials showed that statins, the mainstay of lipid-lowering therapy, reduce LDL-C levels by $30 \%-50 \%$, thereby lowering the relative risk of cardiovascular disease by approximately $30 \%$, regardless of pretreatment LDL-C level. ${ }^{1}$ Nevertheless, in everyday clinical practice, the effectiveness of statins is limited ${ }^{1,3}$ due to noncompliance, discontinuation because of intolerance 
and/or physicians' unawareness of current guidelines, ${ }^{1}$ or a possible suboptimal effect of current therapies. ${ }^{4}$

Emerging therapies may allow for improved lowering of LDL-C over the effects observed with established lipid-lowering agents. For instance, monoclonal antibodies against proprotein convertase subtilisin kexin (PCSK) type 9, a protein involved in LDL-C receptor recycling, are promising new agents with lipid-lowering properties. ${ }^{1,5}$ In randomized trials, a reduction in LDL-C of up to $75 \%$ was observed when these agents were administered as monotherapy or in combination with statins to patients at high risk of cardiovascular disease. ${ }^{1,5}$ It is therefore expected that a greater number of treated patients will attain low $(<1.3 \mathrm{mmol} / \mathrm{L}, \sim 50 \mathrm{mg} / \mathrm{dL})$ or very low ( $<0.65 \mathrm{mmol} / \mathrm{L}, \sim 25 \mathrm{mg} / \mathrm{dL})$ LDL-C levels. ${ }^{5}$ Because cholesterol plays an important role in human physiology, it is plausible that persistently low LDL-C may confer various adverse effects. ${ }^{1}$ A number of studies have reported associations between low LDL-C and increased risk of cancer, ${ }^{2,6-11}$ hemorrhagic stroke, ${ }^{2,12}$ neurodegenerative ${ }^{13}$ and psychiatric diseases, ${ }^{14}$ and non-cardiovascular death. ${ }^{2}$ However, limitations such as potential reverse causation ${ }^{15}$ and use of only single/baseline LDL-C measurements preclude firm conclusions about the risks associated with low LDL-C. ${ }^{8-14,16,17}$ Also, given the effectiveness of currently available lipid-lowering therapies, few patients reach and maintain low or very low levels of LDL-C, which limits the ability to study the impact on health outcomes.

Considering the potential introduction of PCSK type 9 inhibitors, the scarcity of observational data on low LDL-C calls for identification and characterization of patients with low LDL-C in the premarketing environment. Specifically, there is a need for estimates of the background occurrence of events of interest and for ways to identify potential confounders for safety monitoring or comparative effectiveness studies. The objective of the current study was to identify and characterize persons with low LDL-C using data from the Danish medical databases.

\section{Materials and methods Setting}

The Danish National Health Service provides tax-funded medical care for all Danish residents. ${ }^{18}$ Health services provided are registered in various medical databases using the unique Civil Personal Register (CPR) number assigned to all Danish residents by the Civil Registration System since $1968 .{ }^{18}$ Using registry data, we conducted the current study in the North and Central Denmark regions (henceforth collectively called northern Denmark). In the first quarter of
2011, the total adult population of northern Denmark was 1,463,997 persons (source: http://statistikbanken.dk/). The CPR number allowed for exact individual-level linkage of the data from all sources. ${ }^{18}$ All registry codes used in the study are listed in Table S1.

\section{Study population}

We identified all adults with at least one LDL-C measurement (taken after their 18th birthday) recorded in the Clinical Laboratory Information System (LABKA) research database. ${ }^{19}$ The LABKA database collects results from routine tests performed on all specimens submitted by hospitals, general practitioners, and specialists for analysis at hospitalbased laboratories located in northern Denmark. ${ }^{19}$ The database includes the patient's CPR number, the test results, and the date of testing. Types of tests are coded using the International Nomenclature, Properties and Units (NPU) code (http://www.labterm.dk/Enterprise\%20Portal/NPU search. aspx). In addition, for some analyses, a local analysis code is recorded in addition to or instead of the NPU code. Hospitals in the two regions began transferring data gradually to the LABKA database in 1990, reaching complete geographical coverage in 1997 in the North Denmark region and in 2000 in the Central Denmark region. ${ }^{19}$ Based on a crosstabulation of LDL-C measurements and calendar time for each region, registration in the Central Denmark region did not appear as complete as that in the North Denmark region until 2005, when taking their relative population sizes into account (data not shown). To ensure high completeness of the LDL-C data in this analysis, we included measurements recorded between January 1, 1998 and December 31, 2011 in the North Denmark region and between January 1, 2005 and December 31, 2011 in the Central Denmark region. The study population was restricted to persons with uninterrupted residency in the study area during the study period. We considered LDL-C measurements ranging from $>0 \mathrm{mmol} / \mathrm{L}$ to $10 \mathrm{mmol} / \mathrm{L}$ (inclusive); levels outside this range were deemed implausible. The LABKA database may contain records of measurements on the same time and date in one individual; in such situations, we used the mean of the measurements.

\section{Categories of LDL-C levels}

Based on the lowest LDL-C level measured during the study period, we categorized the study population into groups of low LDL-C $(<1.3 \mathrm{mmol} / \mathrm{L}, \sim 50 \mathrm{mg} / \mathrm{dL})$, moderate LDL-C (1.3-3.3 mmol/L, $\sim 50-128 \mathrm{mg} / \mathrm{dL})$, or high LDL-C (>3.3 mmol/L, $128 \mathrm{mg} / \mathrm{dL})$. Within the low LDL-C 
group, we further identified individuals with very low LDL-C $(<0.65 \mathrm{mmol} / \mathrm{L}, \sim 25 \mathrm{mg} / \mathrm{dL})$. The date of the lowest LDL-C measurement during the study period was the index date. Because we were interested in studying LDL-C levels that are rarely seen in clinical practice, but may become more common with the potential introduction of PCSK type 9 inhibitors, ${ }^{20}$ our cutoff defining low LDL-C was lower than

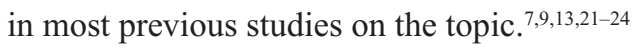

We defined persistence of low LDL-C as a sequence of consecutive measurements, all showing low LDL-C, where at least two measurements in the sequence were $\geq 10$ days apart and no two consecutive measurements were more than 365 days apart. This definition was based on the 2006 Danish guidelines for treatment of dyslipidemia. These guidelines recommend dietary changes as first-line treatment, with assessment of lipid status 1-2.5 months following diet initiation. ${ }^{25}$ Insufficient effect of or compliance with the dietary changes is an indication for addition of lipid-lowering agents followed up with assessment of LDL-C levels within 4-12 weeks after treatment initiation and by checks at least annually once the treatment goal has been achieved. Thus, defining the LDL-C level in a period spanned by two consecutive measurements of low LDL-C, separated by no more than 365 days, as persistently low was deemed sufficiently broad to accommodate any changes in guidelines that may have occurred during the study period. Furthermore, in a sensitivity analysis, we extended the maximum period between two consecutive low LDL-C measurements to 2 years. This definition is likely to capture most repeat measurements, because LDL-C is primarily measured in candidates for receipt of lipid-lowering therapy for secondary prevention, who consult their physician regularly as part of follow-up for their primary disease.

The date for the first low LDL-C measurement in a series of consecutively low LDL-C measurements was considered the index date for the analysis of persistence. Thus, the index date for the persistence analysis may differ from the analysis based on first-time low LDL-C, because some individuals had changing LDL-C levels and did not achieve a persistently low LDL-C level until later in the study period (Figure 1). For persons with several discrete periods of persistently low LDL-C levels separated by a period of higher LDL-C levels, we only considered the first persistently low period.

\section{Patient characteristics}

We used the Danish National Patient Registry (DNPR) ${ }^{26}$ to retrieve patients' history of hospital encounters from birth or DNPR establishment, whichever came last, until and including the index date. The DNPR contains information on all inpatient hospitalizations since 1977 and all hospital specialist outpatient clinic and emergency room visits since 1995. ${ }^{26}$ Each record contains the patient's CPR number, dates of hospital encounters, and one or more discharge diagnoses, including a dedicated field for the primary diagnosis, which represents the main reason for the hospital encounter. Diagnoses were coded according to the International Classification of Diseases, Eighth Revision (ICD-8) during 1977-1993 and the Tenth Revision (ICD-10) thereafter. As a measure of the comorbidity burden, we included the Charlson Comorbidity Index (CCI). ${ }^{27}$ We expressed the severity of comorbidity by using categories constructed based on the CCI total score (low, 0; moderate, 1-2; and severe, $3+$ ) and by tabulating the 19 diseases in the index individually. We also included comorbidities not captured by the CCI, namely, angina pectoris, hypertension, stroke, venous thromboembolism, hospital-diagnosed obesity, chronic kidney disease, hypertensive nephropathy, infection, Parkinson's disease, anxiety, and affective disorders. For myocardial infarction, angina pectoris, congestive heart failure, peripheral vascular disease, cerebrovascular disease, and infection, we examined recent history (events in the 180 days prior to the index date) in addition to history since 1977.

The Aarhus University Prescription Database (AUPD) collects data on all outpatient prescriptions reimbursed to persons in the study area. ${ }^{28}$ All records in the AUPD include the date of dispensing, the patient's CPR number, the Anatomical Therapeutic Chemical code, and the medication name, pack size, dose units, route of administration, and manufacturer. ${ }^{28}$ We used the AUPD to identify current use of the following medications as a measure of underlying disease in the study population: lipid-lowering agents, angiotensin-converting enzyme inhibitors, angiotensin receptor blockers, beta-blockers, calcium channel blockers, diuretics, combination antihypertensive tablets, nitrates, anticoagulants (vitamin $\mathrm{K}$ antagonists, low molecular weight heparin, thrombin inhibitors, and factor Xa inhibitors), antiplatelet drugs (clopidogrel, dipyridamole, and acetylsalicylic acid), antidiabetic drugs (oral and insulin), antidepressants, antipsychotics, antiulcer drugs, systemic glucocorticoids, and bisphosphonates. For all agents, we defined current use as at least one redeemed prescription within 90 days prior to the index date (inclusive).

\section{Statistical analysis}

First, we obtained the distribution of all lowest LDL-C measurements in the study population. We then grouped patients 

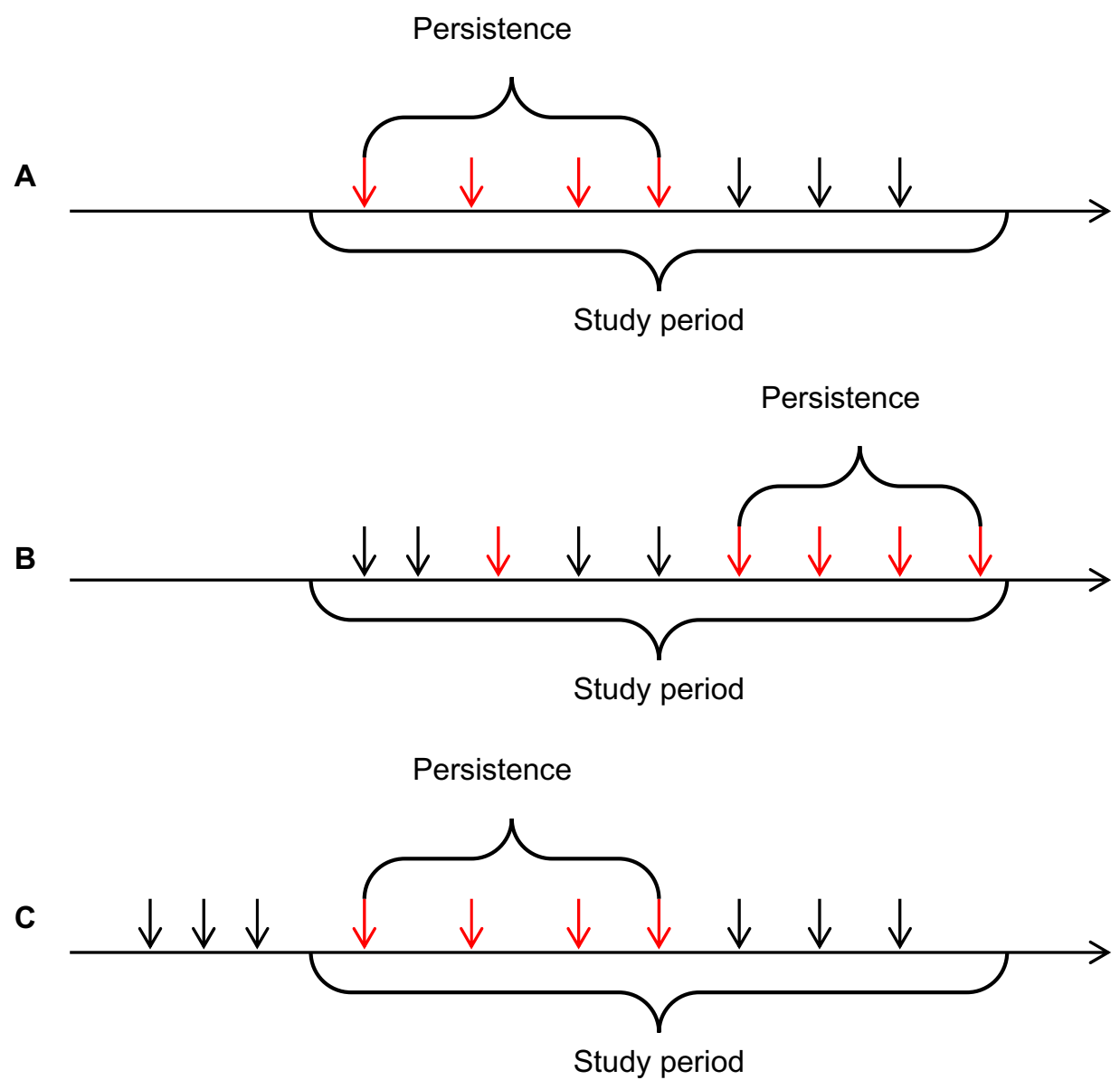

Figure I Illustration of possible situations that may occur in the study given the definitions used. Red arrows mark measurements in the low LDL-C group, whereas black arrows mark measurements at higher LDL-C levels. In (A), the first measurement is low and it is truly the first-ever measurement for this person. The index date for the lowest LDL-C is also the same as in the persistence analysis, because persistence is achieved immediately. In (B), the lowest LDL-C does not correspond to the first measurement during the study period and persistence is not achieved until later because of changing LDL-C level. That is, the index date is different in the persistence analysis in this situation. In (C), the first-ever measurement during the study perid is low and persists at this low level for a period. However, compared with the situation in (A), it is not truly the first-ever measurement due to left censoring.

Abbreviation: LDL-C, low-density lipoprotein cholesterol.

according to the four LDL-C categories and described their demographic and medical characteristics overall and stratified by current use of lipid-lowering agents. For patients identified as having persistently low LDL-C, we recorded the time from the first to the last consecutive observation of low LDL-C, grouped patients according to quartiles of that variable, and cross-tabulated the group characteristics overall and by use of lipid-lowering agents.

The groups in our analysis may represent a mix of individuals with low LDL-C in their first-ever measurement and individuals with a history of higher LDL-C levels prior to achieving a low LDL-C, as illustrated in Figure 1. Furthermore, use of the 90-day definition of current use could misclassify users with longer prescription coverage or suboptimal adherence as non-users of lipid-lowering agents. As a result, patients with low LDL-C would consist of patients with both biological and therapy-induced low
LDL-C levels. We therefore performed a sensitivity analysis, in which we characterized individuals at the time of their first measurement (with missing or non-missing result) during the study period. We restricted this analysis to measurements in 2007 or later to assure at least 2 years of history to assess previous LDL-C measurements. Furthermore, we used the national version of the prescription database ${ }^{29}$ and applied a prescription window of at least 3 years to classify use of lipid-lowering therapy, which should capture more specifically individuals with biological low LDL-C. For other prescription medications, we kept the 90-day prescription window.

Finally, in a sensitivity analysis for the persistence analysis, we allowed the maximum period between two consecutive low LDL-C measurements to extend to 2 years but used the quartile cutoffs from the main analysis to maintain comparability. 
The study was approved by the Danish Data Protection Agency (record number 2013-41-1924). Danish legislation does not require ethical review board approval or informed consent from subjects for registry-based studies.

\section{Results}

\section{Patient characteristics by LDL-C level}

A flowchart for the selection of LDL-C measurements in the study is presented in Figure 2. In total, we identified 5,188,406 LDL-C measurements in the LABKA database. From this, we excluded 990,634 measurements that had missing or implausible results and measurements recorded outside the study period. We then averaged LDL-C results recorded on the same time and date, leaving 3,688,047 measurements. Subsequently, we excluded 24,336 measurements that were registered for pediatric patients, persons with invalid CPR number, and/or persons who had already died or emigrated from Denmark. Finally, 199,751 measurements taken in patients with interrupted residency in the study region were excluded, which left 3,463,960 LDL-C measurements for the study, corresponding to 765,503 persons with at least one LDL-C measurement. The median LDL-C concentration across all measurements was $2.9 \mathrm{mmol} / \mathrm{L}$ (interquartile range $2.2-3.6 \mathrm{mmol} / \mathrm{L}$ ).

There were 172,648 (23\%) persons in the high LDL-C category, 556,077 (73\%) in the moderate LDL-C category, and $36,778(4.8 \%)$ in the low LDL-C category. The latter category included 3,542 ( $0.46 \%$ of total) persons in the very low LDL-C group. Use of lipid-modifying drugs prior to the index date was observed in $2.1 \%$ of patients in the high LDL-C group, $27 \%$ in the moderate LDL-C group, $59 \%$ in the low LDL-C group, and 54\% in the very low LDL-C group. In total, 177,830 persons (89\%) were current users of lipidmodifying drugs prior to their lowest LDL-C value recorded and $12 \%$ of these were in the low LDL-C group.

Compared with patients with moderate or high LDL-C levels, the group with low LDL-C had a higher proportion of men, consisted of older patients, and had a higher prevalence of comorbidities (Table 1). Specifically, there was a higher prevalence of cardiovascular disease, diabetes, chronic

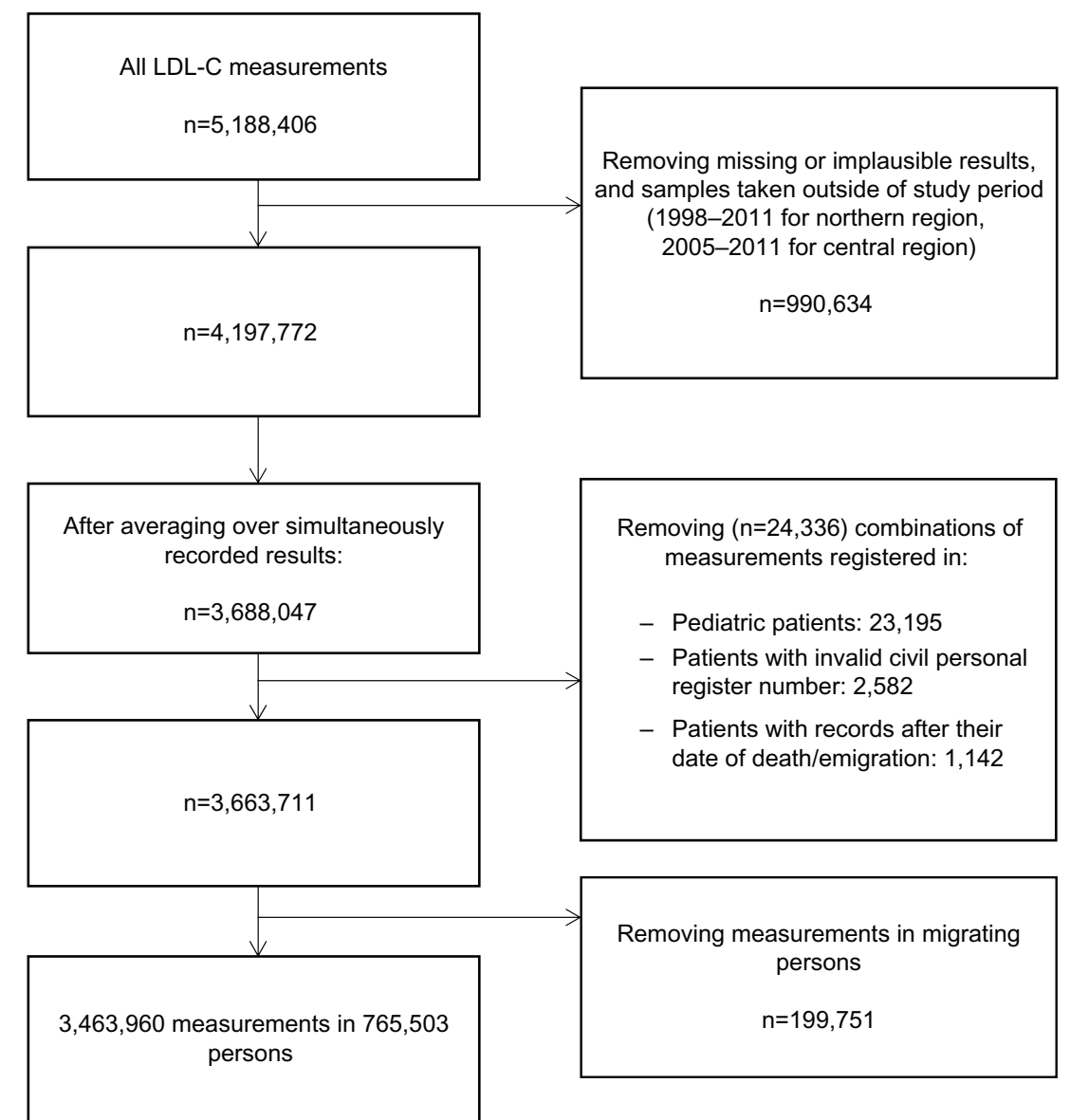

Figure 2 Flowchart for the study.

Abbreviation: LDL-C, low-density lipoprotein cholesterol. 
Table I Characteristics of individuals with an LDL-C measurement in northern Denmark, 1998-20II, by LDL-C level ${ }^{\mathrm{a}}$ and use of lipid-lowering drugs

\begin{tabular}{|c|c|c|c|c|c|}
\hline \multirow[t]{2}{*}{ Characteristic $^{\mathrm{b}, \mathrm{c}}$} & \multicolumn{3}{|l|}{ High LDL-C } & \multicolumn{2}{|l|}{ Moderate LDL-C } \\
\hline & $\begin{array}{l}\text { Lipid-modifying } \\
\text { drug users } \\
\text { n (\%) }\end{array}$ & $\begin{array}{l}\text { Lipid-modifying } \\
\text { drug nonusers } \\
\text { n (\%) }\end{array}$ & $\begin{array}{l}\text { Total } \\
\text { n (\%) }\end{array}$ & $\begin{array}{l}\text { Lipid-modifying } \\
\text { drug users } \\
\text { n (\%) }\end{array}$ & $\begin{array}{l}\text { Lipid-modifying } \\
\text { drug nonusers } \\
\text { n (\%) }\end{array}$ \\
\hline Total & 3,541 & 169,107 & 172,648 & 152,458 & 403,619 \\
\hline \multicolumn{6}{|l|}{ Demographics } \\
\hline Male sex & I,788 (50) & $85,966(5 \mathrm{I})$ & $87,754(5 I)$ & $76,167(50)$ & 187,573 (46) \\
\hline Age, median (interquartile range) & $60(51-69)$ & $55(45-65)$ & $55(45-65)$ & $65(57-73)$ & $53(42-66)$ \\
\hline \multicolumn{6}{|l|}{$\mathrm{CCl}$ score } \\
\hline Low (0) & $2,329(66)$ & I38,545 (82) & $140,874(82)$ & 75,7II (50) & $302,677(75)$ \\
\hline Moderate $(I-2)$ & $944(27)$ & $25,945(15)$ & $26,889(16)$ & $58,974(39)$ & $80,908(20)$ \\
\hline Severe $(3+)$ & $268(8)$ & $4,617(3)$ & $4,885(3)$ & $17,773(12)$ & $20,034(5)$ \\
\hline \multicolumn{6}{|l|}{ Comorbidities } \\
\hline Myocardial infarction & $253(7)$ & $1,690(1)$ & $\mathrm{I}, 943(\mathrm{I})$ & $19,205(13)$ & $8,645(2)$ \\
\hline Congestive heart failure & $116(3)$ & $1,716(1)$ & $\mathrm{I}, 832(\mathrm{I})$ & $7,647(5)$ & $7,769(2)$ \\
\hline Peripheral vascular disease & $192(5)$ & $2,277(I)$ & $2,469(1)$ & II,455 (8) & $9,330(2)$ \\
\hline Cerebrovascular disease & $307(9)$ & $4,54 \mid(3)$ & $4,848(3)$ & $22,017(14)$ & $18,312(5)$ \\
\hline Dementia & $22(1)$ & $627(0)$ & $649(0)$ & $990(1)$ & $\mathrm{I}, 782(0)$ \\
\hline Chronic pulmonary disease & $240(7)$ & $7,807(5)$ & $8,047(5)$ & $12,029(8)$ & $25,031(6)$ \\
\hline Connective tissue disease & $92(3)$ & $3,292(2)$ & $3,384(2)$ & $4,639(3)$ & $9,767(2)$ \\
\hline Ulcer disease & $|3|(4)$ & $3,814(2)$ & $3,945(2)$ & $7,126(5)$ & II,394 (3) \\
\hline Mild liver disease & $25(I)$ & $845(0)$ & $870(1)$ & $\mathrm{I}, 247(\mathrm{I})$ & $3,492(1)$ \\
\hline Diabetes types I and 2 & $114(3)$ & $\mathrm{I}, 409(\mathrm{I})$ & $\mathrm{I}, 523(\mathrm{I})$ & $15,858(10)$ & $|4| ,78(4)$ \\
\hline Hemiplegia & $6(0)$ & $264(0)$ & $270(0)$ & $319(0)$ & $890(0)$ \\
\hline Moderate to severe renal disease & $77(2)$ & $\mathrm{I}, 254(\mathrm{I})$ & $\mathrm{I}, 33 \mathrm{I}(\mathrm{I})$ & $2,949(2)$ & $4,685(I)$ \\
\hline Diabetes with end-organ damage & $53(1)$ & $454(0)$ & $507(0)$ & $6,896(5)$ & $5,396(1)$ \\
\hline Any tumor & $226(6)$ & $8,892(5)$ & $9,118(5)$ & $|2,84|(8)$ & $23,745(6)$ \\
\hline Leukemia & $3(0)$ & $166(0)$ & $169(0)$ & $222(0)$ & $559(0)$ \\
\hline Lymphoma & $22(I)$ & $44 I(0)$ & $463(0)$ & $564(0)$ & $|, 23|(0)$ \\
\hline Moderate to severe liver disease & $2(0)$ & $193(0)$ & $195(0)$ & $242(0)$ & $860(0)$ \\
\hline Metastatic solid tumor & $26(I)$ & $835(0)$ & $861(0)$ & $1,007(\mathrm{I})$ & $2,409(I)$ \\
\hline AIDS & $4(0)$ & $24(0)$ & $28(0)$ & $60(0)$ & $34 I(0)$ \\
\hline
\end{tabular}

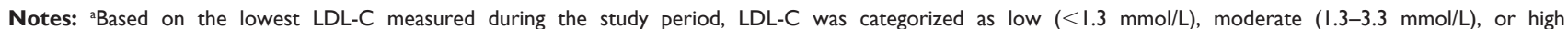
( $>3.3 \mathrm{mmol} / \mathrm{L})$ LDL-C. Very low LDL-C $(<0.65 \mathrm{mmol} / \mathrm{L})$ was defined as a subgroup of low LDL-C; ball characteristics were assessed before or on the date of the lowest LDL-C measurement; 'because of rounding, some percentages are expressed as zero.

Abbreviations: AIDS, acquired immune deficiency syndrome; CCl, Charlson Comorbidity Index; LDL-C, low-density lipoprotein cholesterol.

pulmonary disease, ulcer disease, cancer, hospital-diagnosed obesity, and recent diagnoses of cardiovascular disease and infections (Tables 1 and $\underline{\mathrm{S} 2}$ ). In accordance with the comorbidities observed, use of antihypertensive drugs, nitrates, anticoagulants, antiplatelet drugs, antidiabetic drugs, and antiulcer drugs was more prevalent in the low LDL-C group than in the moderate and high LDL-C groups, as was use of antidepressants and antipsychotics. Despite a comparable age distribution across the LDL-C categories, the differences in patient characteristics became even more pronounced when restricted to patients with very low LDL-C (Tables 1 and $\underline{\text { S2}}$ ). Differences between groups of LDL-C were also observed among current users and non-current users of lipid-modifying drugs (Tables 1 and $\underline{\mathrm{S} 3}$ ). Within each of the LDL-C groups, current users of lipid-modifying drugs were older and had a higher comorbidity burden, with a high prevalence of cardiovascular disease, diabetes, and use of antihypertensive drugs, nitrates, antiplatelet drugs, antiulcer drugs, and antidepressants.

The results from the sensitivity analysis using the firstever LDL-C measurement and a longer prescription window for lipid-lowering agents showed that the group of nonusers with low or very low LDL-C contained a lower proportion of men, were of younger mean age, and had a lower prevalence of most comorbidities and medication use when compared with the main analysis (Table S4). Thus, among persons with low LDL-C, the differences in characteristics between nonusers and users of lipid-lowering agents became even 


\begin{tabular}{|c|c|c|c|c|c|c|}
\hline \multirow[b]{2}{*}{$\begin{array}{l}\text { Total } \\
\text { n (\%) }\end{array}$} & \multicolumn{3}{|l|}{ Low LDL-C } & \multicolumn{3}{|l|}{ Very low LDL-C } \\
\hline & $\begin{array}{l}\text { Lipid-modifying } \\
\text { drug users } \\
\text { n (\%) }\end{array}$ & $\begin{array}{l}\text { Lipid-modifying } \\
\text { drug nonusers } \\
\text { n (\%) }\end{array}$ & $\begin{array}{l}\text { Total } \\
\text { n (\%) }\end{array}$ & $\begin{array}{l}\text { Lipid-modifying } \\
\text { drug users } \\
\text { n (\%) }\end{array}$ & $\begin{array}{l}\text { Lipid-modifying } \\
\text { drug nonusers } \\
\text { n (\%) }\end{array}$ & $\begin{array}{l}\text { Total } \\
\text { n (\%) }\end{array}$ \\
\hline 556,077 & 21,831 & 14,947 & 36,778 & 1,924 & 1,618 & 3,542 \\
\hline $263,740(47)$ & $12,809(59)$ & 8,567 (57) & $21,376(58)$ & I,I85 (62) & $976(60)$ & $2,|6|(6 \mid)$ \\
\hline $58(45-69)$ & $68(60-76)$ & $62(48-74)$ & $66(56-75)$ & $68(59-76)$ & $63(52-74)$ & $66(56-75)$ \\
\hline $378,388(68)$ & $5,834(27)$ & $6,429(43)$ & $12,263(33)$ & 375 (19) & $557(34)$ & $932(26)$ \\
\hline 139,882 (25) & $10,047(46)$ & $5,468(37)$ & $15,515(42)$ & $783(4 I)$ & 633 (39) & $1,416(40)$ \\
\hline $37,807(7)$ & $5,950(27)$ & $3,050(20)$ & $9,000(24)$ & $766(40)$ & $428(26)$ & I, $194(34)$ \\
\hline $27,850(5)$ & $4,4 \mid 4(20)$ & $\mathrm{I}, 432(10)$ & $5,846(16)$ & $424(22)$ & $165(10)$ & $589(17)$ \\
\hline $15,4 \mid 6(3)$ & $2,453(\mathrm{II})$ & $\mathrm{I}, \mathrm{I} 50(8)$ & $3,603(10)$ & $291(15)$ & $132(8)$ & $423(12)$ \\
\hline $20,785(4)$ & $3,130(14)$ & $\mathrm{I}, 32 \mathrm{I}(9)$ & $4,45 \mathrm{I}(12)$ & $386(20)$ & $192(12)$ & $578(16)$ \\
\hline $40,329(7)$ & $4,469(20)$ & I,932 (I3) & $6,401(17)$ & $437(23)$ & $238(15)$ & 675 (19) \\
\hline $2,772(0)$ & $261(1)$ & $122(1)$ & $383(1)$ & $25(I)$ & $16(1)$ & $4 I(I)$ \\
\hline 37,060 (7) & 2,795 (I3) & $\mathrm{I}, 787(\mathrm{I} 2)$ & $4,582(12)$ & $293(15)$ & $261(16)$ & $554(16)$ \\
\hline I4,406 (3) & $822(4)$ & $539(4)$ & $\mathrm{I}, 36 \mathrm{I}(4)$ & $79(4)$ & $81(5)$ & $160(5)$ \\
\hline $18,520(3)$ & I,704 (8) & I, I73 (8) & $2,877(8)$ & $214(\mathrm{II})$ & $189(12)$ & $403(\mathrm{II})$ \\
\hline $4,739(1)$ & $412(2)$ & $577(4)$ & $989(3)$ & $56(3)$ & $10 \mid(6)$ & I57 (4) \\
\hline $30,036(5)$ & $6,367(29)$ & $2,737(18)$ & $9,104(25)$ & $762(40)$ & $325(20)$ & $\mathrm{I}, 087(3 \mathrm{I})$ \\
\hline $\mathrm{I}, 209(0)$ & $78(0)$ & $55(0)$ & $133(0)$ & $8(0)$ & $7(0)$ & $15(0)$ \\
\hline $7,634(\mathrm{I})$ & $\mathrm{I}, 005(5)$ & $646(4)$ & $|, 65|(4)$ & I33 (7) & $99(6)$ & $232(7)$ \\
\hline I2,292 (2) & $3,424(16)$ & $\mathrm{I}, 340(9)$ & $4,764(13)$ & $48 \mid(25)$ & $158(10)$ & $639(18)$ \\
\hline 36,586 (7) & 2,308 (II) & $\mathrm{I}, 490(10)$ & $3,798(10)$ & $227(I 2)$ & $202(12)$ & $429(12)$ \\
\hline $78 \mid(0)$ & $49(0)$ & $75(I)$ & $124(0)$ & $10(1)$ & $9(1)$ & $19(1)$ \\
\hline I,795 (0) & $|2|(1)$ & III (I) & $232(1)$ & $15(I)$ & $22(\mathrm{I})$ & $37(I)$ \\
\hline I,I02 (0) & $81(0)$ & $181(1)$ & $262(1)$ & $14(I)$ & $27(2)$ & $41(I)$ \\
\hline $3,4 I 6(I)$ & $203(I)$ & $187(1)$ & $390(1)$ & $28(I)$ & $26(2)$ & $54(2)$ \\
\hline $401(0)$ & $9(0)$ & $87(\mathrm{I})$ & $96(0)$ & $2(0)$ & $17(I)$ & $19(1)$ \\
\hline
\end{tabular}

larger than was seen in the main analysis. The difference in characteristics when comparing individuals with nonusers of lipid-lowering drugs across LDL-C groups was diminished. The remaining results were not substantially different from the results of the main analysis.

\section{Persistence of low LDL-C}

Among the 36,778 individuals with low LDL-C, 9,612 (44\%) had persistently low LDL-C. The median duration of persistence was 189 days (interquartile range 97-342 days, range 10-2,926 days).

The following characteristics were more prevalent among patients in the lowest quartile compared with the highest quartile of duration of low LDL-C persistence: female sex, severe CCI score, myocardial infarction, congestive heart failure, peripheral vascular disease, ulcer disease, hypertension, and use of nitrates, antidepressants, and antiulcer drugs (Tables 2, S5, and S6). A diagnosis of diabetes or use of antidiabetic drugs were less prevalent. We observed no substantial differences for the remaining characteristics. Stratification by use of lipid-lowering drugs showed the same pattern of variation.

The sensitivity analysis extending the maximum interval between consecutive LDL-C measurements from 1 to 2 years shifted individuals toward the group with the longest persistence. The maximum duration of persistence changed from 2,926 to 3,610 days. Among nonusers of lipid-lowering drugs with the longest duration of low LDL-C, comorbidities and 
Table 2 Characteristics of patients with LDL-C persistently below $1.3 \mathrm{mmol} / \mathrm{L}$, northern Denmark, 1998-20II, by quartiles of time elapsed between first and last consecutively low LDL-C and use of lipid-lowering agents ${ }^{\mathrm{a}}$

\begin{tabular}{|c|c|c|c|c|c|}
\hline \multirow[t]{2}{*}{ Characteristic ${ }^{\mathrm{b}, \mathrm{c}}$} & \multicolumn{3}{|c|}{ First quartile ( $10-96$ days) } & \multicolumn{2}{|c|}{ Second quartile (97-1 89 days) } \\
\hline & $\begin{array}{l}\text { Lipid-modifying } \\
\text { drug users } \\
\text { n (\%) }\end{array}$ & $\begin{array}{l}\text { Lipid-modifying } \\
\text { drug nonusers } \\
\text { n (\%) }\end{array}$ & $\begin{array}{l}\text { Total } \\
\text { n (\%) }\end{array}$ & $\begin{array}{l}\text { Lipid-modifying } \\
\text { drug users } \\
\text { n (\%) }\end{array}$ & $\begin{array}{l}\text { Lipid-modifying } \\
\text { drug nonusers } \\
\text { n (\%) }\end{array}$ \\
\hline Total & 1,690 & 661 & 2,351 & 1,772 & 636 \\
\hline \multicolumn{6}{|l|}{ Demographics } \\
\hline Male sex & $\mathrm{I}, 030(6 \mathrm{I})$ & $415(63)$ & $\mathrm{I}, 445(6 \mathrm{I})$ & $\mathrm{I}, 070(60)$ & $390(61)$ \\
\hline Age, median (interquartile range) & $68(59-76)$ & $64(52-74)$ & $67(57-75)$ & $68(59-75)$ & $66(56-74)$ \\
\hline \multicolumn{6}{|l|}{$\mathrm{CCl}$ score } \\
\hline Low (0) & $38 \mid(23)$ & $205(31)$ & $586(25)$ & $494(28)$ & $206(32)$ \\
\hline Moderate (I-2) & $790(47)$ & $276(42)$ & $1,066(45)$ & $823(46)$ & $264(42)$ \\
\hline Severe $(3+)$ & $519(31)$ & $180(27)$ & $699(30)$ & $455(26)$ & $166(26)$ \\
\hline \multicolumn{6}{|l|}{ Comorbidities } \\
\hline Myocardial infarction & $406(24)$ & $105(16)$ & $5 I I(22)$ & $337(19)$ & $93(15)$ \\
\hline Congestive heart failure & $228(13)$ & $71(11)$ & $299(13)$ & $178(10)$ & $63(10)$ \\
\hline Peripheral vascular disease & $264(16)$ & $76(11)$ & $340(14)$ & $233(13)$ & $71(11)$ \\
\hline Cerebrovascular disease & $336(20)$ & $95(14)$ & $43 \mid(18)$ & 334 (19) & $96(15)$ \\
\hline Dementia & $19(1)$ & $2(0)$ & $21(1)$ & $18(I)$ & $2(0)$ \\
\hline Chronic pulmonary disease & $196(12)$ & $91(14)$ & $287(12)$ & $228(13)$ & $66(10)$ \\
\hline Connective tissue disease & $68(4)$ & $22(3)$ & $90(4)$ & $65(4)$ & $34(5)$ \\
\hline Ulcer disease & $152(9)$ & $66(10)$ & $218(9)$ & $150(8)$ & $57(9)$ \\
\hline Mild liver disease & $30(2)$ & $28(4)$ & $58(2)$ & $4 I(2)$ & $28(4)$ \\
\hline Diabetes types I and 2 & $56 \mid(33)$ & $179(27)$ & $740(31)$ & $597(34)$ & $155(24)$ \\
\hline Hemiplegia & $6(0)$ & $4(1)$ & $10(0)$ & $4(0)$ & $4(1)$ \\
\hline Moderate to severe renal disease & $100(6)$ & $39(6)$ & $139(6)$ & $75(4)$ & $34(5)$ \\
\hline Diabetes with end-organ damage & $318(19)$ & III (I7) & $429(18)$ & $300(17)$ & $74(12)$ \\
\hline Any tumor & $165(10)$ & $63(10)$ & $228(10)$ & $138(8)$ & $64(10)$ \\
\hline Leukemia & $8(0)$ & $4(1)$ & $12(1)$ & $4(0)$ & $\mathrm{I}(0)$ \\
\hline Lymphoma & $5(0)$ & II (2) & $16(I)$ & $7(0)$ & $2(0)$ \\
\hline Moderate to severe liver disease & $8(0)$ & $10(2)$ & $18(I)$ & $7(0)$ & $5(1)$ \\
\hline Metastatic solid tumor & $14(I)$ & $7(1)$ & $21(I)$ & $9(1)$ & $6(1)$ \\
\hline AIDS & $2(0)$ & $9(1)$ & II (0) & $0(0)$ & $13(2)$ \\
\hline
\end{tabular}

Notes: ${ }^{a}$ We defined as consecutive any two LDL-C measurements that were at least 10 days and no more than 365 days apart; ball characteristics were assessed before or on the date for the first low LDL-C measurement in a series of consecutively low LDL-C; cbecause of rounding, some percentages are expressed as zero.

Abbreviations: AIDS, acquired immune deficiency syndrome; $\mathrm{CCI}$, Charlson Comorbidity Index; LDL-C, low-density lipoprotein cholesterol.

medication use were less frequent than in the main analysis (Table S7). Apart from this finding, no substantial differences in patient characteristics were observed when compared with the main analysis.

\section{Discussion}

In this study, we identified 765,503 persons with at least one LDL-C measurement and found a prevalence of $4.8 \%$ for having ever had a low LDL-C and $0.46 \%$ for having ever had a very low LDL-C. Persons with low LDL-C were more often users of lipid-lowering agents and had a greater comorbidity burden than those with higher LDL-C. In particular, cardiovascular disease and diabetes, as well as use of prescription drugs for treatment of these diseases, were more prevalent. This finding reflects that, in Denmark, lipid-lowering therapy is primarily used as secondary prevention for patients with cardiovascular disease and as primary prevention for high-risk patients, eg, patients with type 2 diabetes, type 1 diabetes with microalbuminuria, or an estimated risk of cardiovascular death of $\geq 5 \%$ within 10 years according to the Systematic Coronary Risk Evaluation. ${ }^{25}$ However, the same patterns were present, albeit less pronounced, when comparing persons who had no prescription history of lipid-lowering agents. It is possible that these persons achieved their low LDL-C through lifestyle changes or as a consequence of other acute or chronic comorbid conditions. Persons with low LDL-C had a higher prevalence of recent cardiovascular disease or infection, which may reflect suppression of cholesterol levels due to the acute phase reaction in association with the recent illness. ${ }^{30}$ However, it may also reflect the effect of recent initiation on lipid-lowering therapy as secondary prevention, and the prevalence of recent diagnoses were not particularly high among nonusers. 


\begin{tabular}{|c|c|c|c|c|c|c|}
\hline \multirow[b]{2}{*}{$\begin{array}{l}\text { Total } \\
\text { n (\%) }\end{array}$} & \multicolumn{3}{|c|}{ Third quartile ( $190-342$ days) } & \multicolumn{3}{|c|}{ Fourth quartile (343-2,926 days) } \\
\hline & $\begin{array}{l}\text { Lipid-modifying } \\
\text { drug users } \\
n(\%)\end{array}$ & $\begin{array}{l}\text { Lipid-modifying } \\
\text { drug nonusers } \\
n(\%)\end{array}$ & $\begin{array}{l}\text { Total } \\
\text { n (\%) }\end{array}$ & $\begin{array}{l}\text { Lipid-modifying } \\
\text { drug users } \\
\text { n (\%) }\end{array}$ & $\begin{array}{l}\text { Lipid-modifying } \\
\text { drug nonusers } \\
\text { n (\%) }\end{array}$ & $\begin{array}{l}\text { Total } \\
\text { n (\%) }\end{array}$ \\
\hline 2,408 & $\mathrm{I}, 734$ & 714 & 2,448 & I,792 & 613 & 2,405 \\
\hline $\mathrm{I}, 460(6 \mathrm{I})$ & $\mathrm{I}, 062(6 \mathrm{I})$ & $445(62)$ & $\mathrm{I}, 507(62)$ & $\mathrm{I}, \mathrm{I} 44(64)$ & $390(64)$ & $\mathrm{I}, 534(64)$ \\
\hline $67(59-75)$ & $68(60-76)$ & $65(55-74)$ & $67(59-75)$ & $68(60-74)$ & $64(56-74)$ & $67(59-74)$ \\
\hline $700(29)$ & $446(26)$ & $246(34)$ & $692(28)$ & $477(27)$ & $222(36)$ & $699(29)$ \\
\hline $\mathrm{I}, 087(45)$ & $831(48)$ & $294(4 I)$ & $\mathrm{I}, \mathrm{I} 25(46)$ & $875(49)$ & $248(40)$ & $\mathrm{I}, \mathrm{I} 23(47)$ \\
\hline $621(26)$ & $457(26)$ & $174(24)$ & $631(26)$ & $440(25)$ & $143(23)$ & $583(24)$ \\
\hline $430(18)$ & 333 (19) & $97(14)$ & $430(18)$ & 343 (19) & $81(13)$ & $424(18)$ \\
\hline $24 I(10)$ & $205(12)$ & $52(7)$ & $257(10)$ & $162(9)$ & $48(8)$ & $210(9)$ \\
\hline $304(13)$ & $225(13)$ & $87(12)$ & $312(13)$ & $239(13)$ & $52(8)$ & 291 (I2) \\
\hline 430 (18) & 34 I (20) & $106(15)$ & 447 (I8) & 333 (19) & $79(13)$ & $412(17)$ \\
\hline $20(1)$ & $10(1)$ & $2(0)$ & $12(0)$ & $9(1)$ & $3(0)$ & $12(0)$ \\
\hline 294 (I2) & $218(13)$ & $93(13)$ & $311(13)$ & $167(9)$ & $64(10)$ & $231(10)$ \\
\hline 99 (4) & $72(4)$ & $16(2)$ & $88(4)$ & $52(3)$ & $19(3)$ & 7I (3) \\
\hline 207 (9) & $120(7)$ & $59(8)$ & $179(7)$ & $114(6)$ & $42(7)$ & $156(6)$ \\
\hline $69(3)$ & $45(3)$ & $36(5)$ & 81 (3) & $30(2)$ & $25(4)$ & $55(2)$ \\
\hline $752(31)$ & $552(32)$ & $211(30)$ & $763(3 \mathrm{I})$ & $632(35)$ & $184(30)$ & $816(34)$ \\
\hline $8(0)$ & $6(0)$ & I (0) & $7(0)$ & $9(1)$ & $0(0)$ & $9(0)$ \\
\hline $109(5)$ & $68(4)$ & $40(5)$ & $108(4)$ & $72(4)$ & $31(5)$ & $103(4)$ \\
\hline $374(16)$ & $302(17)$ & $107(15)$ & 409 (I7) & $326(18)$ & $95(15)$ & 421 (I8) \\
\hline 202 (8) & I50 (9) & 78 (II) & $228(9)$ & 157 (9) & $52(8)$ & 209 (9) \\
\hline $5(0)$ & $2(0)$ & $3(0)$ & $5(0)$ & $3(0)$ & $3(0)$ & $6(0)$ \\
\hline $9(0)$ & $10(1)$ & I (0) & II (0) & $4(0)$ & $7(1)$ & II (0) \\
\hline $12(0)$ & $5(0)$ & $8(1)$ & $13(I)$ & $6(0)$ & $5(1)$ & II (0) \\
\hline $15(1)$ & $9(1)$ & $6(1)$ & $15(I)$ & $6(0)$ & $7(1)$ & $13(1)$ \\
\hline $13(1)$ & $0(0)$ & $5(1)$ & $5(0)$ & $0(0)$ & $6(1)$ & $6(0)$ \\
\hline
\end{tabular}

To our knowledge, only few studies have described the characteristics of patients with LDL-C $<1.3 \mathrm{mmol} / \mathrm{L} .^{31-33}$ In a post hoc analysis of the JUPITER trial, those attaining an LDL-C $<50 \mathrm{mg} / \mathrm{dL}(\sim 1.3 \mathrm{mmol} / \mathrm{L})$ were more likely to be male, white, and to have impaired fasting glucose than those with higher LDL-C levels. ${ }^{31}$ Among 2,099 patients with acute myocardial infarction or non-ST-segment elevation who were randomized to $80 \mathrm{mg}$ atorvastatin (versus $40 \mathrm{mg}$ pravastatin) in the PROVE IT-TIMI 22 study, 9.5\% reached an LDL-C level $<40 \mathrm{mg} / \mathrm{dL}(\sim 1.03 \mathrm{mmol} / \mathrm{L})$ within 4 months. $^{32}$ These patients were more likely to be older, male, to have diabetes, and to have a lower baseline LDL$\mathrm{C}$, but less likely to have had a prior myocardial infarction or coronary artery bypass graft, to smoke, or to have used statins within 2 weeks before study initiation, as compared with those achieving an LDL-C $>80-100 \mathrm{mg} / \mathrm{dL}$. These earlier studies were based on data from randomized clinical trials ${ }^{31,32}$ in which populations are likely to be more selected (eg, inclusion of only persons without cardiovascular disease and diabetes and with LDL-C levels $<130 \mathrm{mg} / \mathrm{dL}$ in the JUPITER trial' ${ }^{31}$ ) than in our study including a broad population-based sample of persons who all had their LDL-C measured at least once. Indeed, our study is more comparable with a large Korean hospital-based study of patients with LDL-C $\leq 40 \mathrm{mg} / \mathrm{dL}$ showing a similar prevalence of cardiovascular disease, diabetes, cancer, statin use, and antiplatelet drug use. ${ }^{33}$ However, fewer than $1 \%$ of all patients with an LDL-C measurement had LDL-C $\leq 40 \mathrm{mg} / \mathrm{dL}$ compared with $9.6 \%$ with LDL-C $\leq 50 \mathrm{mg} / \mathrm{dL}$ in our study, ${ }^{33}$ which may be explained by differences in ethnicity or setting (inclusion of general practice also in our study). Although several other studies have characterized study subjects by LDL-C levels, they are not comparable with ours because the limit defining a low LDL-C level (varied 
between 1.81 to $2.65 \mathrm{mmol} / \mathrm{L}$ ) corresponded to a moderate LDL-C level in our study. 7,9,13,21-24

Because information in the LABKA database is recorded electronically to provide an easily accessible diagnostic tool for health care providers, we expected a low level of missing data for performed laboratory tests. However, our cross-tabulation of LDL-C measurements and calendar period indicated periods with suboptimal completeness. To optimize completeness, we excluded measurements from calendar years in which completeness was judged insufficient. Only a small proportion of the LDL-C measurements had implausible values, but erroneous measurements that fall within the range of plausible values may have caused misclassification between LDL-C subgroups. Finally, although we included all persons with an LDL-C test recorded (approximately 50\% of residents), inclusion was most likely selective based on indications for performing an LDL-C test (eg, risk evaluation for atherosclerotic cardiovascular disease and treatment with drugs that may affect lipoprotein metabolism). A US study based on insurance claims data found that among 703,484 patients initiating lipid-lowering treatment, LDL-C tests were indeed performed selectively. ${ }^{34}$ For example, recent hospitalization and myocardial infarction were negatively associated with having a test performed. ${ }^{34}$ However, the findings cannot be extrapolated to our setting because the US study included only outpatient laboratory tests and because health care system characteristics differ.

Patients with high LDL-C who survive to achieve a low LDL-C measurement may represent a selected group of patients with some favorable lifestyle characteristics that outweigh the risks of having a high LDL-C level. This selection could explain why persons with low LDL-C were older and in turn had a higher comorbidity burden. Furthermore, some patients with high LDL-C may die of competing risks before ever having their LDL-C measured. Thus, we may have selected patients with non-fatal cardiovascular events, potentially related to the LDL-C level. Similarly, selection bias due to differences in survival may have been introduced in the persistence analysis. It is possible that some persons have a short duration of low LDL-C merely because they die before having their low LDL-C measurement repeated. Thus, individuals with persistent low LDL-C may represent a selected group of survivors.

The positive predictive values for many diagnoses identified in the DNPR are generally high, but vary depending on the gold standard against which they are compared and by the prevalence of disease and therefore department of origin. ${ }^{35-41}$ Confirmed against hospital medical records for the diagnoses assigned by the treating physician, the overall positive predictive value of the CCI categories is $98 \%{ }^{42}$ Because the DNPR does not reflect diagnoses made in primary care, completeness for conditions typically diagnosed and/or treated in hospital settings (eg, myocardial infarction, stroke, and cancer) is likely to be higher than for conditions treated by general practitioners. The prevalence of hospital diagnoses for diseases such as chronic pulmonary disease, hypertension, and diabetes may thus be underestimated in our study. However, we compensated for the lack of diagnostic data from primary care by including information on prescriptions reimbursed for disease-specific drugs. ${ }^{43}$

\section{Conclusion}

In light of the new promising lipid-lowering agents emerging on the drug market, the proportion of treated persons achieving low or very low LDL-C levels is expected to grow. ${ }^{5}$ However, the potential risks and benefits associated with such low levels are yet to be established..$^{5}$ In this study, we identified 36,778 persons with low LDL-C, among whom 3,542 had very low LDL-C. Compared with persons with higher LDL-C levels, persons with low LDL-C were older, were more often users of lipid-lowering agents, and had a greater comorbidity burden, particularly cardiovascular disease and diabetes. The large group of persons with low and very low LDL-C identified in this study also demonstrates the feasibility of future longitudinal studies examining background rates of cardiovascular and non-cardiovascular diseases in persons with low LDL-C.

\section{Disclosure}

This study was partially supported by an unrestricted grant from Amgen Inc. issued to and administered by Aarhus University. AM is employed at Amgen Inc. SAJS, UHJ, and VE are salaried employees of Aarhus University or Aarhus University Hospital and receive no direct financial compensation from Amgen Inc. or any other pharmaceutical company. The authors report no other conflicts of interest in this work.

\section{References}

1. Besseling J, van Capelleveen J, Kastelein JJP, Hovingh GK. LDL cholesterol goals in high-risk patients: how low do we go and how do we get there? Drugs. 2013;73(4):293-301.

2. LaRosa JC, Pedersen TR, Somaratne R, Wasserman SM. Safety and effect of very low levels of low-density lipoprotein cholesterol on cardiovascular events. Am J Cardiol. 2013;111(8):1221-1229.

3. Kanstrup H, Lassen JF, Heickendorff L, Lauritzen T, Larsen ML. Quality of lipid-lowering therapy in patients with ischaemic heart disease: a register-based study in 3477 patients. J Intern Med. 2004;255(3): 367-372.

4. Gitt AK, Drexel H, Feely J, et al. Persistent lipid abnormalities in statin-treated patients and predictors of LDL-cholesterol goal achievement in clinical practice in Europe and Canada. Eur J Prev Cardiol. 2012;19(2):221-230. 
5. Farnier M. PCSK9: From discovery to therapeutic applications. Arch Cardiovasc Dis. 2014;107(1):58-66.

6. Alsheikh-Ali AA, Trikalinos TA, Kent DM, Karas RH. Statins, lowdensity lipoprotein cholesterol, and risk of cancer. $J$ Am Coll Cardiol. 2008;52(14):1141-1147.

7. Benn M, Tybjærg-Hansen A, Stender S, Frikke-Schmidt R, Nordestgaard BG. Low-density lipoprotein cholesterol and the risk of cancer: a Mendelian randomization study. J Natl Cancer Inst. 2011;103(6):508-519.

8. Fiorenza AM, Branchi A, Sommariva D. Serum lipoprotein profile in patients with cancer. A comparison with non-cancer subjects. Int J Clin Lab Res. 2014;30(3):141-145.

9. Shor R, Wainstein J, Oz D, et al. Low serum LDL cholesterol levels and the risk of fever, sepsis, and malignancy. Ann Clin Lab Sci. 2007;37(4): 343-348.

10. Swanson CA, Potischman N, Barrett RJ, et al. Endometrial cancer risk in relation to serum lipids and lipoprotein levels. Cancer Epidemiol Biomarkers Prev. 1994;3(7):575-581.

11. Yang X, So W, Ko GTC, et al. Independent associations between lowdensity lipoprotein cholesterol and cancer among patients with type 2 diabetes mellitus. CMAJ. 2008;179(5):427-437.

12. Wang X, Dong Y, Qi X, Huang C, Hou L. Cholesterol levels and risk of hemorrhagic stroke: a systematic review and meta-analysis. Stroke 2013;44(7):1833-1839.

13. Huang X, Abbott RD, Petrovitch H, Mailman RB, Ross GW. Low LDL cholesterol and increased risk of Parkinson's disease: prospective results from Honolulu-Asia Aging Study. Mov Disord. 2008;23(7): 1013-1018.

14. Troisi A. Cholesterol in coronary heart disease and psychiatric disorders: same or opposite effects on morbidity risk? Neurosci Biobehav Rev. 2009;33(2):125-132.

15. Strasak AM, Pfeiffer RM, Brant LJ, et al. Time-dependent association of total serum cholesterol and cancer incidence in a cohort of 172,210 men and women: a prospective 19-year follow-up study. Ann Oncol. 2009;20(6):1113-1120.

16. Yang X, So WY, Ma RCW, et al. Low LDL cholesterol, albuminuria, and statins for the risk of cancer in type 2 diabetes: the Hong Kong Diabetes Registry. Diabetes Care. 2009;32(10):1826-1832.

17. Wulaningsih W, Garmo H, Holmberg L, et al. Serum Lipids and the Risk of Gastrointestinal Malignancies in the Swedish AMORIS Study. J Cancer Epidemiol. 2012;2012:792034.

18. Schmidt M, Pedersen L, Sørensen HT. The Danish Civil Registration system as a tool in epidemiology. Eur J Epidemiol. 2014;29(8): 541-549.

19. Grann AF, Erichsen R, Nielsen AG, Frøslev T, Thomsen RW. Existing data sources for clinical epidemiology: the clinical laboratory information system (LABKA) research database at Aarhus University, Denmark. Clin Epidemiol. 2011;3:133-138.

20. Robinson JG, Nedergaard BS, Rogers WJ, et al. Effect of evolocumab or ezetimibe added to moderate- or high-intensity statin therapy on LDL-C lowering in patients with hypercholesterolemia: the LAPLACE-2 randomized clinical trial. JAMA. 2014;311(18):1870-1882.

21. Noda H, Iso H, Irie F, et al. Low-density lipoprotein cholesterol concentrations and death due to intraparenchymal hemorrhage: the Ibaraki Prefectural Health Study. Circulation. 2009;119(16):2136-2145.

22. Imamura T, Doi Y, Arima H, et al. LDL cholesterol and the development of stroke subtypes and coronary heart disease in a general Japanese population: the Hisayama study. Stroke. 2009;40(2):382-388.

23. Charach G, George J, Roth A, et al. Baseline low-density lipoprotein cholesterol levels and outcome in patients with heart failure. Am J Cardiol. 2010;105(1):100-104.

24. Kim BJ, Lee S-H, Ryu W-S, Kang BS, Kim CK, Yoon B-W. Low level of low-density lipoprotein cholesterol increases hemorrhagic transformation in large artery atherothrombosis but not in cardioembolism. Stroke. 2009;40(5):1627-1632.
25. Institute for Rational Pharmacotherapy. [Guidelines for lipid-lowering therapy]. Available from: http://www.irf.dk/dk/publikationer/rationel_ farmakoterapi/maanedsblad/2006/lipidsaenkende_behandling.htm. Accessed April 9, 2014. Danish.

26. Lynge E, Sandegaard JL, Rebolj M. The Danish National Patient Register. Scand J Public Health. 2011;39(7 Suppl):30-33.

27. Charlson ME, Pompei P, Ales KL, MacKenzie CR. A new method of classifying prognostic comorbidity in longitudinal studies: development and validation. J Chronic Dis. 1987;40(5):373-383.

28. Ehrenstein V, Antonsen S, Pedersen L. Existing data sources for clinical epidemiology: Aarhus University Prescription Database. Clin Epidemiol. 2010;2:273-279.

29. Johannesdottir SA, Horvath-Puho E, Ehrenstein V, Schmidt M, Pedersen L, Sørensen HT. Existing data sources for clinical epidemiology: The Danish National Database of Reimbursed Prescriptions. Clin Epidemiol. 2012;4:303-313

30. Balci B. The modification of serum lipids after acute coronary syndrome and importance in clinical practice. Curr Cardiol Rev. 2011;7(4): 272-276.

31. Hsia J, MacFadyen JG, Monyak J, Ridker PM. Cardiovascular event reduction and adverse events among subjects attaining low-density lipoprotein cholesterol. J Am Coll Cardiol. 2011;57(16):1666-1675.

32. Wiviott SD, Cannon CP, Morrow DA, et al. Can low-density lipoprotein be too low? The safety and efficacy of achieving very low lowdensity lipoprotein with intensive statin therapy: a PROVE IT-TIMI 22 substudy. J Am Coll Cardiol. 2005;46(8):1411-1416.

33. Lee J-G, Koh SJ, Yoo SY, et al. Characteristics of subjects with very low serum low-density lipoprotein cholesterol and the risk for intracerebral hemorrhage. Korean J Intern Med. 2012;27(3):317-326.

34. Schneeweiss S, Rassen JA, Glynn RJ, et al. Supplementing claims data with outpatient laboratory test results to improve confounding adjustment in effectiveness studies of lipid-lowering treatments. BMC Med Res Methodol. 2012;12:180.

35. Madsen M, Davidsen M, Rasmussen S, Abildstrom SZ, Osler M. The validity of the diagnosis of acute myocardial infarction in routine statistics: a comparison of mortality and hospital discharge data with the Danish MONICA registry. J Clin Epidemiol. 2003;56(2):124-130.

36. Phung TKT, Andersen BB, Høgh P, Kessing LV, Mortensen PB, Waldemar G. Validity of dementia diagnoses in the Danish hospital registers. Dement Geriatr Cogn Disord. 2006;24(3):220-228.

37. Obel N, Reinholdt H, Omland LH, Engsig F, Sørensen HT, Hansen A-B. Retrievability in The Danish National Hospital Registry of HIV and hepatitis $\mathrm{B}$ and $\mathrm{C}$ coinfection diagnoses of patients managed in HIV centers 1995-2004. BMC Med Res Methodol. 2008;8:25.

38. Vestberg K, Thulstrup AM, Sørensen HT, Ottesen P, Sabroe S, Vilstrup H. Data quality of administratively collected hospital discharge data for liver cirrhosis epidemiology. J Med Syst. 1997;21(1):11-20.

39. Pedersen M, Klarlund M, Jacobsen S, Svendsen AJ, Frisch M. Validity of rheumatoid arthritis diagnoses in the Danish National Patient Registry. Eur J Epidemiol. 2003;19(12):1097-1103.

40. Johnsen SP, Overvad K, Sørensen HT, Tjønneland A, Husted SE. Predictive value of stroke and transient ischemic attack discharge diagnoses in The Danish National Registry of Patients. $J$ Clin Epidemiol. 2002;55(6):602-607.

41. Nielsen GL, Sørensen HT, Pedersen AB, Sabroe S. Analyses of data quality in registries concerning diabetes mellitus - a comparison between a population based hospital discharge and an insulin prescription registry. J Med Syst. 1996;20(1):1-10.

42. Thygesen SK, Christiansen CF, Christensen S, Lash TL, Sorensen HT. The predictive value of ICD-10 diagnostic coding used to assess Charlson comorbidity index conditions in the population-based Danish National Registry of Patients. BMC Med Res Methodol. 2011; 11(1):83.

43. Demming A. Monitoring medicine: Denmark, an epidemiologist's dream. Projects. 2013;31:122-123. 


\section{Publish your work in this journal}

Clinical Epidemiology is an international, peer-reviewed, open access, online journal focusing on disease and drug epidemiology, identification of risk factors and screening procedures to develop optimal preventative initiatives and programs. Specific topics include: diagnosis, prognosis, treatment, screening, prevention, risk factor modification,

Submit your manuscript here: http://www.dovepress.com/clinical-epidemiology-journal systematic reviews, risk \& safety of medical interventions, epidemiology \& biostatistical methods, and evaluation of guidelines, translational medicine, health policies \& economic evaluations. The manuscript management system is completely online and includes a very quick and fair peer-review system, which is all easy to use. 